\title{
Effect of radial artery harvest on collateral forearm blood flow and digital perfusion
}

\author{
Richard F. Brodman, MD \\ Laurie E. Hirsh, MD \\ Rosemary Frame, FNP, MS
}

From the Department of Cardiothorcic Surgery, Joan and Sanford I. Weill Medical College of Cornell University, New YorkPresbyterian Hospital, New York, NY.

Received for publication May 17, 2001; revisions requested July 13, 2001; revisions received July 18, 2001; accepted for publication July 24, 2001.

Address for reprints: Richard Brodman, MD, Department of Cardiothoracic Surgery, Weill Medical College, 525 East 68th St, New York, NY 10021 (E-mail: rfb20002@mail.med. cornell.edu).

J Thorac Cardiovasc Surg 2002;123:512-6

Copyright () 2002 by The American Association for Thoracic Surgery

$0022-5223 / 2002 \$ 35.00+0 \quad \mathbf{1 2 / 1 / 1 1 9 8 8 5}$

doi:10.1067/mtc.2002.119885
Background: Changes describing digital and forearm circulation after radial artery harvest have been reported infrequently.

Methods: This prospective study examined digital perfusion and forearm collateral circulation preoperatively and postoperatively in patients who underwent coronary artery bypass grafting with radial artery free grafts. Noninvasive evaluation was conducted with digital photoelectric plethysmography and color flow and pulsed Doppler studies.

Results: Thumb perfusion index decreased from 1.25 to $0.84(30 \%, P<.001)$ in the unoperated extremities and from 1.23 to $0.80(36 \%)$ in the operated extremities $(P$ $<.001$ ). Doppler studies in extremities after radial artery harvest demonstrated an increase in ulnar artery diameter from 3.87 to $4.66 \mathrm{~mm}(15.7 \%, P<.001)$ and a rise in ulnar blood flow velocity from 38.96 to $48.46 \mathrm{~cm} / \mathrm{s}(17.4 \%)$ preoperatively to 8 weeks postoperatively $(P<.001)$. No hand ischemia was noted.

Conclusions: Our study identified a mild reduction in digital perfusion and an increase in ulnar artery flow velocity and diameter with no clinical sequelae or compromise in hand function after radial artery harvest in properly selected patients.

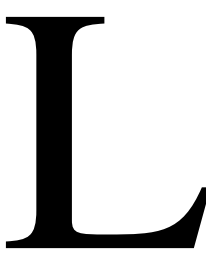

ittle information has appeared in the literature describing changes in forearm and digital circulation after radial artery (RA) harvest. ${ }^{1-3}$ Revival of RA use by Acar and associates ${ }^{4}$ has elicited a resurgence in the use of the RA as a bypass conduit. In the current surgical era there has been one report of ischemia of the hand. This case was reported twice. ${ }^{5,6}$ Fear of development of inadequate collateral circulation may limit harvesting of the RA. Inadequate collateral circulation can result in complications, such as sensitivity to cold-induced reduced grip strength, claudication, digital ulcerative lesions, and ultimately amputation as a result of arterial insufficiency. ${ }^{7-10}$ Accordingly, to determine the short-term effects of the loss of the RA's contribution to hand perfusion, we prospectively examined quantitative and qualitative forearm and digital circulation preoperatively and postoperatively in patients who underwent coronary artery bypass grafting with RA conduits.

\section{Subjects and Methods}

Informed consent for RA harvest was obtained preoperatively from all patients. A total of 389 patients were evaluated for RA harvest, with 305 (78\%) patients undergoing RA harvest. At the time of hospital discharge, follow-up procedures used in this protocol were explained to all patients who underwent RA harvest. Operated arms were defined as those upper extremities from which the RA was harvested; unoperated arms were defined as those upper extremities from which the RA was not harvested. Seventy-eight patients who underwent bilateral harvesting were included in this study. RA harvest was performed by the senior author (R.F.B.). 


\section{Evaluation Procedures}

Preoperative testing performed on the first consecutive 100 patients in this study who had coronary artery bypass grafting with an RA free graft included photoelectric plethysmography with a calculated perfusion index (PI; modified Biox model 3700 pulse oximeter; Datex-Ohmeda, Louisville, Colo) to determine whether adequate collateral circulation was present and color flow and Doppler studies to prospectively evaluate changes in the collateral vessels within the forearm before and after RA harvest. The remaining 289 patients underwent screening for adequate collateral circulation alone by using the PI studies. At the initiation of the study, RA harvest was from the nondominant arm. Therefore, only this arm was evaluated. After the 25 th patient, if the study result was positive (indicating inadequate collateral flow), the dominant arm was evaluated, and if adequate collateral circulation was present, the dominant arm was used for RA harvest. After the 60th patient to undergo RA harvest, the PI was performed, preoperatively and postoperatively, on both upper extremities. Thus, 26 (13\%) patients with unilateral harvest included in this study did not have a PI generated for their unoperated arms.

\section{Timing of Studies}

Postoperative testing duplicated the patients' preoperative testing protocol. Color flow and pulsed Doppler scanning (Acuson 128 Cardiovascular System; Acuson, Inc, Mountainview, Calif) of the RAs and ulnar arteries (UAs) was also performed. Preoperative testing, regardless of the studies performed, took place at any time before the surgical procedure but in general was performed 1 to 3 days before the operation. Postoperatively, photoelectric plethysmography was performed approximately 4 to 5 weeks after the operation, whereas postoperative Doppler evaluations were scheduled 8 to 10 weeks after surgical intervention.

\section{Description of Studies Performed}

Photoelectric plethysmography yielding both an oxygen saturation $\left(\mathrm{SpO}_{2}\right)$ and a calculated PI was performed with a modified pulse oximeter. ${ }^{11}$ The modified oximeter's digital probe was applied to the thumb, ensuring that the photoelectric sensor was correctly positioned over the nail bed, and a blood pressure cuff was applied to the arm. A baseline reading of the $\mathrm{SpO}_{2}$ and the perfusion to the thumbnail bed was obtained. The RA was located by means of palpation, and the blood pressure cuff was inflated to $200 \mathrm{~mm} \mathrm{Hg}$ until the PI indicated approximately zero flow. The RA was compressed manually at the wrist, blood pressure cuff inflation was released, and compression of the RA was maintained for a period of approximately 45 seconds. Flow to the hand with RA occlusion is through the UA. After 45 seconds, the PI and $\mathrm{SpO}_{2}$ levels were recorded. The compression of the RA was released, the UA was located at the wrist, the blood pressure cuff was reinflated to 200 $\mathrm{mm} \mathrm{Hg}$, and UA was compressed. After 45 seconds, the PI and $\mathrm{SpO}_{2}$ were recorded. The procedure was then repeated on the contralateral arm. This protocol was used in an effort to duplicate somewhat what happens during the modified Allen test, interrupting blood flow to the hand, but our protocol does not require patient cooperation and does not disturb the digital sensor. The ratio of the PI with the RA occluded and the PI with no occlusion (baseline) was used as an index of blood flow to the hand independent of RA flow; this is the perfusion index ratio (PIR). The
PIR with the RA occluded is indicative of the flow primarily through the UA. The criterion we used for a negative study indicating that RA harvesting would be safe was a ratio of $40 \%$ or greater. ${ }^{12,13}$ Preoperative studies evaluated the contribution of both the RA and UA to thumb perfusion and $\mathrm{SpO}_{2}$. In the operated arms postoperatively, only the UA contribution could be assessed, whereas in the unoperated arms, both the contribution of the RA and that of the UA could be assessed.

Color flow and pulsed Doppler scanning were used to determine both the lumen diameter and flow velocities, respectively. The Doppler studies were performed by a $7.5-\mathrm{MHz}$ phased array transducer. The Doppler beam, ranging from 3 to $10 \mathrm{MHz}$, is emitted from a ceramic crystal in the transducer head. The distal RAs and UAs were bilaterally evaluated for internal diameter and flow velocities at a point just proximal to the wrist crease. Cardiac monitor dots (Red Dot, model 2239; 3M Company, Medical Device Division, St Paul, Minn) were applied to the patient, and the patient was connected to the electrocardiography monitor of the Doppler machine. All recordings were made in systole as noted by the onset of the QRS complex from the electrocardiography tracings. Aqueous gel (Aquasonic 100; Aquasonic Parker Laboratory, Orange, NJ) was applied on the transducer head, and the probe was positioned at a $60^{\circ}$ angle to the volar aspect of the forearm. Once the arterial signal was detected, the image was frozen on screen, and the diameter was calculated with internal calipers. Flow was determined by relocating the artery and toggling the pulse-velocity switch. Internal calipers were used to measure the flow velocities obtained. Preoperative studies evaluated both the RAs and UAs bilaterally. Postoperatively, the UA diameter and flow velocity were calculated in the operated arm or arms, and both the RA and UA diameters and flows were obtained in the unoperated arms.

Postoperative clinical evaluation included examination for compromise in hand function, including strength, grip, ability to perform fine motor function, and hand ischemia.

Analysis of the data was performed with SPSS for Windows, version 6.1 (SPSS, Inc, Chicago, Ill). Means, SDs, and ranges were calculated. Data were compared with paired $t$ tests. The unoperated arms from patients with unilateral RA harvest served as controls.

\section{Results}

Of the 193 patients who had both preoperative and postoperative testing, there were $151(78.2 \%)$ male and 42 $(21.85 \%)$ female patients. Seventy-eight $(40.4 \%)$ patients underwent bilateral RA harvest. The mean \pm SD age was $60.27 \pm 10.52$ years (range, $1-88$ years); mean ejection fraction was $58.89 \% \pm 14.27 \%$ (range, $10 \%-85 \%$ ). There was a mean of $3.27 \pm 0.95$ bypass grafts per patient, with 1.61 of these grafts fashioned from the RA. Mean body surface area was $1.94 \pm 0.2 \mathrm{~m}^{2}$. Mean cardiopulmonary bypass time was $90.45 \pm 35.14$ minutes; mean crossclamp time was $58.84 \pm$ 26.21 minutes.

There was a statistically significant increase in the preoperative to postoperative $\mathrm{SpO}_{2}$ in the unoperated extremities, from $95.09 \%$ to $96.13 \%(\mathrm{n}=98, P<.001)$, and a corresponding statistically significant change in the operated arms 
TABLE 1. Perfusion index

\begin{tabular}{lcccc}
\hline & Preoperative PI $(\mathbf{n}=\mathbf{8 7})$ & Postoperative PI $(\mathbf{n}=\mathbf{1 1 5})$ & Pvalues & PIR \\
\hline Unoperated extremities (range) & $1.25 \pm 0.72(0.08-3.7)$ & $0.84 \pm 0.46(0.08-2.8)$ & $<.001$ & $0.65 \pm 0.29$ \\
Operated extremities (range) & $1.23 \pm 0.72(0.14-1.3)$ & $0.80 \pm 0.46(0.08-2.6)$ & $<.001$ & $0.77 \pm 0.23$ \\
$P$ values & .767 & .252 & .001
\end{tabular}

TABLE 2. Distal RA hemodynamics: Color flow and pulsed Doppler findings

\begin{tabular}{|c|c|c|c|c|c|c|}
\hline & $\begin{array}{l}\text { Preoperative diameter, } \\
\qquad n=46(\mathrm{~mm})\end{array}$ & $\begin{array}{l}\text { Postoperative diameter, } \\
\qquad n=21(\mathrm{~mm})\end{array}$ & $P$ value & $\begin{array}{l}\text { Preoperative velocity, } \\
\qquad n=47(\mathrm{~cm} / \mathrm{s})\end{array}$ & $\begin{array}{l}\text { Postoperative velocity, } \\
\qquad n=47 \text { (mm) }\end{array}$ & $P$ value \\
\hline Unoperated arms (range) & $4.29 \pm 1.22(2.0-8.6)$ & $4.72 \pm 1.2(2.4-8.6)$ & .185 & $37.58 \pm 8.98(14-61)$ & $40.28 \pm 11$ & .193 \\
\hline Operated arms & $4.02 \pm 0.96(2.4-8.6)$ & & & $36.66 \pm 11.55$ & & \\
\hline$P$ values & .173 & & & .47 & & \\
\hline
\end{tabular}

TABLE 3. Distal UA hemodynamics: Color flow and pulsed Doppler findings

\begin{tabular}{|c|c|c|c|c|c|c|}
\hline & $\begin{array}{l}\text { Preoperative diameter, } \\
\quad n=46(\mathrm{~mm})\end{array}$ & $\begin{array}{l}\text { Postoperative diameter, } \\
n=41(\mathrm{~mm})\end{array}$ & $P$ values & $\begin{array}{l}\text { Preoperative velocity, } \\
\quad \mathrm{n}=41(\mathrm{~cm} / \mathrm{s})\end{array}$ & $\begin{array}{c}\text { Postoperative velocity, } \\
n=41(\mathrm{~mm})\end{array}$ & $P$ value \\
\hline Unoperated arms & 4.01 & 3.9 & .616 & 38.39 & 41.78 & .254 \\
\hline Operated arms & 3.87 & 4.66 & $<.001$ & 38.96 & 48.46 & $<.001$ \\
\hline$P$ values & .939 & .001 & & .727 & .308 & \\
\hline
\end{tabular}

of $94.99 \%$ to $95.78 \%$ ( $\mathrm{n}=223, P<.001)$. There was no significant difference in the baseline preoperative $\mathrm{SpO}_{2}$ or in the postoperative values between unoperated and operated arms $(\mathrm{n}=110)$. These statistical differences do not translate into clinical significance because all saturations, preoperatively and postoperatively, in both operated and unoperated arms, are within the normal physiologic range of $92 \%$ or greater.

The results for the PI are summarized in Table 1. There was no difference in the baseline preoperative PI of the unoperated and operated extremities $(n=87)$ and in the postoperative baseline flows $(n=115)$. A significant difference existed between the calculated preoperative PIRs during RA occlusion between the unoperated and operated extremities, measuring 0.65 and 0.77 , respectively $(P=.001)$, when all patients evaluated were included. However, when the value for the unoperated patients who had positive results are removed, there is no difference in the baseline ratios during RA occlusion (PIR of 0.75 for the unoperated arms and 0.76 for the operated arms, $P=.658$ ). There was no difference in the PIR during UA occlusion $(P=653)$ between the unoperated extremities preoperatively to postoperatively $(P=.083)$. A significant decline in the PI was noted in both the unoperated and operated arms after the operation $(P \leq .001$ for both): a $30.4 \%$ decline in the unoperated arms $(\mathrm{n}=80)$ and a $6 \%$ further decline in the operated arms $(n=258)$, which represents the loss of the RA flow to the hand. This further decline in RA perfusion was not significant $(P=.252)$.
Results of the color flow Doppler scanning demonstrated that there was no significant difference between the baseline preoperative diameters of either the RAs or UAs in 46 unoperated and operated arms $(P=.173$, Tables 2 and 3$)$. There was no significant preoperative to postoperative change in the diameter of the 21 RAs in the unoperated extremities. Given that the RA was harvested from the operated arms, this value could not be assessed postoperatively. Measurement of the distal UA diameter revealed no significant changes when comparing preoperative to postoperative size in the unoperated arms. However, there was a significant increase preoperatively to postoperatively in the 41 operated extremities evaluated, from 3.87 to $4.66 \mathrm{~mm}(P=.001)$. This difference was significantly increased compared with the UA's diameter in the unoperated arms $(P=.001)$.

There was no statistical difference in the baseline preoperative RA flow velocities among the 47 unoperated arms. There was no change in either the RA or UA velocities in the unoperated arms preoperatively to postoperatively. Corresponding to the significant increase in UA diameter, UA velocity was found to significantly increase preoperatively to postoperatively in the 41 operated arms, from 38.96 to $48.46 \mathrm{~cm} / \mathrm{s}(P<.001)$. This difference was not significantly different when compared with the unoperated extremities $(P=.308)$.

Qualitative assessment of forearm and digital perfusion revealed no evidence of ischemia. Two patients reported 
mild sensitivity to extremely cold temperatures (approximately $0^{\circ} \mathrm{F}-20^{\circ} \mathrm{F}$ ) during the winter; the sensitivity diminished by wearing warmer gloves. After RA harvest, there was no loss of fine or gross motor function, nor did patients have functional limitations. Ten patients reported transient dysesthesias in operated extremities along the distribution of the superficial branch of the radial nerve or the lateral antebrachial cutaneous nerve, which are not vascular in cause; all resolved spontaneously and completely without further sequelae.

\section{Discussion}

Vascular supply to the forearm is provided by an anastomotic network, receiving contributing branches from the $\mathrm{UA}, \mathrm{RA}$, and anterior and posterior interosseous arteries. Patterns of dominant flow through the hand varies. Hirai $^{14,15}$ found that $26 \%$ of the population he studied had radial dominant circulation, $24 \%$ were ulnar dominant, and $50 \%$ showed no dominant vessel, whereas Fuhrman and colleagues $^{16}$ found that $28 \%$ of his 88 patients were radial dominant, $44 \%$ were ulnar dominant, $24 \%$ were indeterminate dominant, and $4 \%$ demonstrated an interrupted arch. Knowledge of the dominant vessel in one upper extremity does not predict the dominance in the contralateral side. ${ }^{16-}$ 18 A physiologically complete palmar arch system and unobstructed flow through the UA are essential prerequisites for RA harvest. The anatomy of the palmar arch system need not be verified. The functionality of the collateral network must be objectively and reproducibly assessed. Preoperative assessment must be performed to verify the presence of adequate collateral forearm circulation and to identify individuals who would have inadequate perfusion to the hand if deprived of RA blood flow.

There has been unsubstantiated trepidation in the harvesting of the RA for fear of ischemic sequelae in the hand, sensitivity to cold temperature, or development of hand claudication. In the current surgical era, the issue of arterial insufficiency caused by a loss of radial blood flow has been addressed in the context of RA cannulation and radial forearm flaps. ${ }^{7-9,19}$ Analogous to physiologic compensatory changes seen in other parts of the vascular system when vascular occlusion occurs as a result of thrombi or stenosis, the growth of the UA's diameter and flow velocity can be postulated to occur to compensate for the loss of the RA's contribution to the hand's blood supply. A few studies evaluated the effect of RA harvest on digital and UA blood flow preoperatively and postoperatively. ${ }^{1-3}$ These studies demonstrate that compensatory changes in flow occur to maintain adequate hand circulation in selected patients.

Our own study used photoelectric plethysmography, providing both $\mathrm{SpO}_{2}$ and a measure of digital blood flow. Pulse oximetry determines the amount of hemoglobin $\mathrm{SpO}_{2}$ by comparing the differences in light absorbed by oxygenated and unoxygenated hemoglobin. ${ }^{20}$ The normal range for $\mathrm{SpO}_{2}$ is $92 \%$ or greater. Pulse oximetry with an unmodified pulse oximeter alone is not adequate to measure flow; that is, the pulse oximeter simply measures the amount of oxygenated hemoglobin passing through its sensor. Hemoglobin may remain saturated at flows consistent with ischemia that are inadequate to support circulation to the hand. ${ }^{19,21-23}$ In our study all mean values for $\mathrm{Spo}_{2}$ were within physiologic parameters, and no clinical significance corresponds to saturations of greater than $92 \%$. Therefore, the slight increase in $\mathrm{SpO}_{2}$ noted postoperatively was merely a statistical observation. In 31 patients who had a positive PIR test result preoperatively (demonstrating inadequate collateral circulation), ${ }^{24}$ an $\mathrm{SpO}_{2}$ of $92 \%$ or greater during RA occlusion was maintained. Two patients had saturations of less than $92 \%$ at baseline that did not further drop during RA compression. Of interest, in these 31 patients 20 had normal Allen tests results, 7 patients had positive Allen test results, and the test could not be performed in 4 patients. The results of the Allen test had a weak negative correlation that was not significant compared with the PIR with the RA occluded ( $r=-0.2338, P=.241)$.

The modified pulse oximeter used in our study is capable of assessing digital flow by using the principle of photoelectric plethysmography. PI correlates with digital blood flow with venous occlusion plethysmography $(r=0.94) .{ }^{11}$ The PI is obtained by using a modified pulse oximeter; a standard oximeter finger probe is applied over the nail bed. PI is determined by the change in the path of infrared light emitted from the sensor through the digit in both systole and diastole; by measuring the varying intensity, the change in the path length can be calculated. Greater blood flow into the digit being scanned corresponds to a greater PI. ${ }^{11}$ In this study postoperative PI measurements performed at rest declined significantly in both the operated and unoperated extremities. There was a $33 \%$ decline in the unoperated extremities and an additional $6 \%$ decrease in operated arms, which we believe represents the loss of the RA to the thumb's circulation. It must be noted that the PI, like oximetry, is subject to variation because of changes associated with vasoconstriction induced by ambient temperature and sympathetic tone reflecting changes in the physiologic state. ${ }^{20,23,24}$ Venous congestion, as in cardiac failure, carbon monoxide concentration (typical in cigarette smokers), tachyarrhythmias, and anemia have also been shown to influence these measurements. ${ }^{20}$ Therefore, the drop in the absolute values noted by the baseline flows may represent factors other than decline in digital perfusion. The further drop in the operated to the unoperated extremities cannot be fully explained by these factors except for the surgical removal of the RA. The integrity of the PI as a determinant of the UA's contribution to digital blood flow is demonstrated by lack of difference between the ratio obtained in 
the unoperated arms during RA occlusion before (PIR = 0.66 ) and after surgical intervention (PIR $=0.61, P=.139$ ).

Ultrasound Doppler studies can not only provide measures of vessel diameter and blood flow velocity but can also determine the patency of the RA-palmar artery-UA circuit during contralateral vessel occlusion. ${ }^{1-3,25-29}$ The Doppler studies performed as part of this study were used to quantify the short-term effects of the loss of the RA on the contralateral vessel diameter and in flow to the hand and not to determine the patency of the collateral circuit. In our study, color flow Doppler scanning revealed an $11 \%$ increase in UA diameter, preoperatively to postoperatively, in the arms from which the RA was harvested. UA blood flow velocity, as measured with pulsed Doppler scanning, increased by about $20 \%$ postoperatively. Our findings are consistent with those of Mulvany and associates ${ }^{30}$ and Pola and colleagues, ${ }^{1}$ who found that increasing flow appears to be a powerful stimulus to growth, resulting in an increased lumen.

This noninvasive study prospectively examined the forearm and digital circulation in 386 upper extremities in patients who underwent coronary artery bypass grafting with RA conduits. A significant increase in UA diameter and blood flow velocity 8 weeks after RA harvest was noted. Thumbnail $\mathrm{SpO}_{2}$ remained within physiologic parameters with a slight, nonstatistically significant decline in the PI of the thumb of the arms in which the RA was harvested compared with the arms in which the RA has not been harvested. Clinically, blood flow remains adequate to meet the physiologic demands of the upper extremity without causing hand ischemia or functional limitations. The infrequently noted (3.7\% of the operated forearms) transient dysethesias are not vascular in origin because they occurred in the distribution of the lateral antebrachial cutaneous nerve or the superficial branch of the radial nerve, both of which are vulnerable during harvest. Thus, the compensatory mechanisms available to maintain blood flow and perfusion appear adequate in the short term after RA harvest in properly selected patients to sustain arterial inflow to the hand without clinical functional limitations in those extremities deemed to have forearm and hand collateral circulation by preoperative assessment with photoelectric plethysmography.

\section{References}

1. Pola P, Serricchio M, Flore R, Manasse E, Favuzzi A, Possati GF. Safe removal of the radial artery for myocardial revascularization: a Doppler study to prevent ischemic complications to the hand. $J$ Thorac Cardiovasc Surg. 1996;112:737-44.

2. Dumanian GA, Segelman K, Mispireta LA, Walsh JA, Hendrickson MF, Wilgis EF. Radial artery use in bypass grafting does not change digital blood flow or hand function. Ann Thorac Surg. 1998;65:1284-7.

3. Lohr JM, Paget DS, Smith JM, Winkler JL, Wladis AR. Upper extremity hemodynamic changes after radial artery harvest for coronary artery bypass grafting. Ann Vasc Surg. 2000;14:56-62.

4. Acar C, Jebara VA, Portoghese M, Beyssen B, Pagny JY, Grare P, et al. Revival of the radial artery for coronary artery bypass grafting. Ann Thorac Surg. 1992;54:652-9.
5. Nunoo-Mensah J. An unexpected complication after harvesting of the radial artery coronary artery bypass grafting. Ann Thorac Surg. 1998;66:929-31.

6. Fox AD, Whiteley MS, Phillips-Hughes J, Roake J. Acute upper limb ischemia: a complication of coronary artery bypass grafting. Ann Thorac Surg. 1999;67:535-7.

7. Meland NB, Core GB, Hoverman VR. The radial forearm flap donor site: Should we vein graft the artery? A comparative study. Plast Reconstr Surg. 1993;91:865-70.

8. Suominen S, Ahovuo J, Asko-Seljavaara S. Donor site morbidity of radial forearm flaps: a clinical and ultrasonographic evaluation. Scand J Plast Reconstr Surg Hand Surg. 1996;30:57-61.

9. Toschka H, Feifel H, Erli HJ, Minkenberg R, Paar O, Riediger D. Aesthetic and functional results of harvesting radial forearm flap, especially with regard to hand function. Int J Oral Maxillofac Surg. 2001;30:42-8.

10. Coltharp WH. Revival of the radial artery bypass grafting. Ann Thorac Surg. 1992;54:659-60.

11. Ozaki M, Lopez M. Pulse oximeter-based flow index correlates well with fingertip volume plethysmography [abstract]. Anesthesia. 1993;793:A542.

12. Husum B, Berthelsen P. Allen's test and systolic arterial pressure in the thumb. Br J Anaeth. 1981;53:635-7.

13. Carter S. The definition of critical ischaemia of the lower limb and distal systolic pressures. Br J Surg. 1973;16:111-21.

14. Hirai M. Arterial insufficiency of the hand evaluated by digital blood pressure and arteriographic findings. Circulation. 1978;58:902-8.

15. Hirai M. Digital blood pressure and arteriographic findings under selective compression of the radial and ulnar arteries. Angiology. 1980;31:21-31.

16. Fuhrman TM, Pippin WD, Talmage LA, Reilley TE. Evaluation of collateral circulation of the hand. J Clin Monit. 1992;8:28-32.

17. Fuhrman TM, Reilley TE, Pippin WD. Comparison of digital blood pressure, plethysmography, and the modified Allen's test as means of evaluating the collateral circulation to the hand. Anaesthesia. 1992;47:959-61

18. Gelberman RH, Blasingame JP. The timed Allen test. J Trauma. 1981;21:477-9.

19. Pillow K, Herrick IA. Pulse oximetry compared with Doppler ultrasound for assessment of collateral blood flow to the hand. Anaesthesia. 1991;46:388-90.

20. Griffiths D, Ilsley A, Runciman W. Pulse meters and pulse oximeters. Anaesth Intensive Care. 1988;16:49-53.

21. Fuhrman TM, McSweeney E. Noninvasive evaluation of the collateral circulation to the hand. Acad Emerg Med. 1995;2:195-9.

22. Lawson D, Norley I, Korbon G, Loeb R, Ellis J. Blood flow limits and pulse oximeter signal detection. Anesthesiology. 1987;67:599-603.

23. Levinsohn DG, Gordon L, Sessler DI. The Allen's test: analysis of four methods. J Hand Surg [Am]. 1991;16:279-82.

24. Cheng E, Lauer K, Stommel K, Guenther N. Evaluation of the palmar circulation by pulse oximetry. J Clin Monit. 1989;5:1-3.

25. Yoa ST, Gourmas C, Papathanasiou K, Irvine W. A method for assessing ischemia of the hand and fingers. Surg Gynecol Obstet. 1972;135:373-8

26. Starnes SL, Wolk SW, Lampman RM, Shanley CJ, Prager RL, Kong BK, et al. Noninvasive evaluation of hand circulation before radial artery harvest for coronary artery bypass grafting. $J$ Thorac Cardiovasc Surg. 1999;117:261-6.

27. Hosono M, Suehiro S, Shibata T, Sasaki Y, Kumano H, Kinoshita H. Duplex scanning to assess radial artery suitability for coronary artery bypass grafting. Jpn J Thorac Cardiovasc Surg. 2000;48:217-21.

28. Ruengsakulrach P, Brooks M, Hare DL, Gordon I, Buxton BF. Preoperative assessment of hand circulation by means of Doppler ultrasonography and modified Allen test. $J$ Thorac Cardiovasc Surg. 2001;121:526-31.

29. Rodriguez E, Ormont ML, Lambert EH, Needleman L, Halpern EJ, Diehl JT, et al. The role of preoperative radial artery ultrasound and digital plethysmography prior to coronary artery bypass grafting. Eur J Cardiothorac Surg. 2001;19:135-9.

30. Mulvany MJ, Baumbach GL, Aalkjaer C, Heagerty AM, Korsgaard N, Schiffrin EL, et al. Vascular remodeling. Hypertension. 1996;28:505-6. 